\title{
Violation of causality in $f(T)$ gravity
}

\author{
G. Otalora ${ }^{1, a}$, M. J. Rebouças ${ }^{2, b}$ \\ ${ }^{1}$ Instituto de Física, Pontificia Universidad Católica de Valparaíso, Casilla 4950, Valparaiso, Chile \\ ${ }^{2}$ Centro Brasileiro de Pesquisas Físicas, Rua Dr. Xavier Sigaud 150, Rio de Janeiro, RJ 22290-180, Brazil
}

Received: 24 August 2017 / Accepted: 3 November 2017 / Published online: 23 November 2017

(C) The Author(s) 2017. This article is an open access publication

\begin{abstract}
In the standard formulation, the $f(T)$ field equations are not invariant under local Lorentz transformations, and thus the theory does not inherit the causal structure of special relativity. Actually, even locally violation of causality can occur in this formulation of $f(T)$ gravity. A locally Lorentz covariant $f(T)$ gravity theory has been devised recently, and this local causality problem seems to have been overcome. The non-locality question, however, is left open. If gravitation is to be described by this covariant $f(T)$ gravity theory there are a number of issues that ought to be examined in its context, including the question as to whether its field equations allow homogeneous Gödel-type solutions, which necessarily leads to violation of causality on non-local scale. Here, to look into the potentialities and difficulties of the covariant $f(T)$ theories, we examine whether they admit Gödel-type solutions. We take a combination of a perfect fluid with electromagnetic plus a scalar field as source, and determine a general Gödel-type solution, which contains special solutions in which the essential parameter of Gödel-type geometries, $m^{2}$, defines any class of homogeneous Gödeltype geometries. We show that solutions of the trigonometric and linear classes $\left(m^{2}<0\right.$ and $\left.m=0\right)$ are permitted only for the combined matter sources with an electromagnetic field matter component. We extended to the context of covariant $f(T)$ gravity a theorem which ensures that any perfect-fluid homogeneous Gödel-type solution defines the same set of Gödel tetrads $h_{A}^{\mu}$ up to a Lorentz transformation. We also showed that the single massless scalar field generates Gödeltype solution with no closed time-like curves. Even though the covariant $f(T)$ gravity restores Lorentz covariance of the field equations and the local validity of the causality principle, the bare existence of the Gödel-type solutions makes apparent that the covariant formulation of $f(T)$ gravity does not preclude non-local violation of causality in the form of closed time-like curves.
\end{abstract}

\footnotetext{
a e-mail: giovanni.otalora@pucv.cl

be-mail: reboucas@cbpf.br
}

\section{Introduction}

The frameworks proposed to account for the observed latetime accelerated expansion of the Universe can be roughly grouped into two broad families. In the first, the unknown form of matter sources, the so-called dark energy, is invoked and the underlying gravity theory, general relativity (GR), is kept unmodified. In this framework, the simplest way to describe the accelerated expansion of the Universe is by introducing a cosmological constant, $\Lambda$, into the general relativity field equations. This approach is entirely consistent with the available observational data, but it faces difficulties related to the order of magnitude of the cosmological constant and its microphysical origin. In the second family, modifications of Einstein's field equations are taken as an alternative for describing the late-time accelerated expansion. Examples in this class include generalized gravity theories based upon modifications of the Einstein-Hilbert action by taking nonlinear functions $f(R)$ of the Ricci scalar $R$ or other curvature invariants (for reviews see, e.g., Refs. [1-7]).

Another family of modified gravity theories that has been examined as an alternative way of describing the late-time acceleration of the Universe [8-11] is known as $f(T)$ gravity. In close analogy with the $f(R)$, the $f(T)$ gravity theory was suggested by extending the Lagrangian of teleparallel gravity to a function $f(T)$ of a torsion scalar $T[8,9]$. The Lagrangian density in these so-called teleparallel gravity (TG) theories contains a function $f(T)$ of the torsion scalar $T$ in a formal analogy with $f(R)$ gravity. ${ }^{1}$ In the teleparallel gravity theories the dynamical variables are the tetrad fields $\mathbf{h}_{A}\left(x^{\mu}\right)$ instead of the metric $g_{\mu \nu}$, and rather than the usual torsionless Levi-Civita connection of GR, a Weitzenböck connection, which has torsion but no curvature, is employed to define the covariant derivative. In this way, the gravitational field in

\footnotetext{
1 The very beginning of this subject goes back to Einstein's attempt to unify gravity and electromagnetism in 1928 through the introduction of a vierbein (tetrad) field together with the notion of distant parallelism or teleparallelism $[12,13]$.
} 
teleparallel gravity is described in terms of the torsion instead of the curvature [14-20].

In comparison with $f(R)$ in the metric formalism, whose field equations are of fourth order, the $f(T)$ gravity theories have the advantage that their dynamics is given by secondorder differential equations. This important characteristic, along with the fact that $f(T)$ theories can be used to explain the observed accelerating expansion, has given birth to a fair number of articles on these gravity theories, in which several features of $f(T)$ gravity have been examined, including observational solar system constraints [21-23], cosmological constraints [24-28], cosmological perturbations [29-33], spherically symmetric solutions [34-36], the existence of relativistic stars [37], cosmographic constraints [38] and energy condition bounds [39]. ${ }^{2}$

Despite this noticeable interest in the new gravity theory, it has been pointed out that the field equations of $f(T)$ theory are not invariant under local Lorentz transformations $[62,63]$ unless the $f(T)$ gravity is teleparallel equivalent to general relativity (TEGR), in which $f(T)=\lambda T+\Lambda$, where $\lambda$ and $\Lambda$ are constants. This means that apart from the TEGR, these theories are, in general, sensitive to the choice of the tetradsa Lorentz transformation of the tetrad basis changes the $f(T)$ field equations [64-66]. This problem appears to have been initially overlooked in most of the recent literature on $f(T)$ gravity, but it has recently been considered in Ref. [67], where it has been found that lack of Lorentz invariance arises as a consequence of a particular choice of the spin connection borrowed from TEGR [67]. Relaxing this original choice of the spin connection, a locally Lorentz covariant $f(T)$ gravity theory has then been devised [67].

In special relativity chronology and causality are ingredients so essential that they are simply built into the theory from the very outset-chronology is preserved and causality is respected in the theory. General relativity (GR) inherits locally a chronology protection, which ensures the local validation of the causality principle, from the bare fact that the space-times of general relativity are locally Minkowskian. On a non-local scale, however, important differences may emerge since Einstein's field equations do not provide in general non-local constraints on the underlying space-time manifold. The model found by Gödel [68] is a well-known solution to Einstein's equations that makes it clear that GR admits solutions with closed time-like world lines, despite its local invariance under Lorentz transformations, which ensures the validity of the causality principle locally. ${ }^{3}$

\footnotetext{
${ }^{2}$ For further references on several aspects of $f(T)$ gravity we refer the reader to the review article [11] and the references therein. An incomplete list of more recent references is [40-61].

${ }^{3}$ Gödel's solution has a recognizable importance and has motivated a fair number of investigations on rotating Gödel-type models in the context of general relativity (see, e.g. Refs. [69-76]) and in the framework of other gravity theories (see, for example, Refs. [77-91]).
}

Even though different choices of tetrads related by a Lorentz transformation leave the metric invariant, suggesting at first sight a well-defined local causal structure, in the usual or standard formulation of $f(T)$ every different Lorentz tetrads give rise to different field equations, and therefore represents a different theory. In this way, for a given (fixed) frame of tetrads, which determines a given (fixed) $f(T)$ theory, there is no Lorentz transformation freedom. This means that unlike general relativity, a $f(T)$ gravity theory does not inherit locally a chronology protection from the special relativity, and one may even have a local violation of causality. ${ }^{4}$ This local causality problem seems to have been overcome in the Lorentz covariant $f(T)$ gravity theory [67], since in this new formulation of the theory the Lorentz transformations do not change the metric nor the field equations. A question that naturally arises here is whether the covariant formulation of $f(T)$ gravity allows for Gödel-type solutions, which necessarily lead to non-local violation of the causality principle in the form of closed time-like curves, or would remedy this causal pathology by ruling out this type of solutions, which are permitted in general relativity. Moreover, if gravitation is to be described by this Lorentz covariant $f(T)$ theory [67] there are a number of issues that ought to be reexamined in its context, including the question as to whether these gravity theories allow for non-causal solutions for physically wellbehaved matter sources.

In this paper, we proceed with further investigations on the difficulties, limitations and potentialities of the Lorentz covariant $f(T)$ theory [67], by addressing this question and examining whether this gravity theory admits homogeneous Gödel-type solutions for a quite general matter source. To this end, we take a combination of a perfect fluid with electromagnetic plus scalar fields as a matter source, and determine a general Gödel-type solution of the covariant $f(T)$ field equations. This general solution contains several special solutions, including perfect-fluid and single scalar field solutions. It emerges from our results that this general solution contains special solutions whose essential parameter, $m$, of Gödeltype geometries is positive (hyperbolic family), null (linear family) and negative (trigonometric class). Solutions in the trigonometric class only exist for a combination of sources that includes an electromagnetic field as a matter component. We show that any perfect-fluid ST-homogeneous Gödel-type solution of the covariant $f(T)$ gravity is isometric to the Gödel metric, and therefore exhibits violation of causality. ${ }^{5}$ We also show that a single massless scalar field generates the

\footnotetext{
4 The local violation of causality is also related to an ill-defined Cauchy problem in $\mathrm{f}(\mathrm{T})$ gravity. These issues have been discussed in Refs. [9294].

5 This extends the Bampi-Zordan theorem [96], which states that every perfect-fluid Gödel-type solution to the Einstein field equations is necessarily isometric to the Gödel space-time, to the context of covariant $f(T)$ gravity.
} 
only ST-homogeneous Gödel-type solution without violation of causality of Gödel type. We underline that the bare existence of these ST-homogeneous Gödel-type solutions makes apparent that the Lorentz covariant $f(T)$ gravity does not remedy the causal anomaly in the form of closed time-like curves that are present in general relativity.

The structure of the paper is as follows. In Sect. 2, to define the notation and make this paper to a certain extent self-contained, we give a brief account of the Lorentz covariant $f(T)$ gravity. In Sect. 3 we present the main properties of space-time (ST) homogeneous Gödel-type geometries. This includes the metric, the ST-homogeneity conditions, the non-isometric ST-homogeneous Gödel-type classes, and the existence of closed time-like curves in these space-times. In Sect. 4 we show that the Lorentz covariant $f(T)$ gravity theories admit ST-homogeneous Gödel-type solutions for several physically well-motivated matter contents, and therefore despite the local Lorentz invariance, it houses a non-local violation of causality. In Sect. 5 we present our main conclusions and final remarks.

\section{Covariant $f(T)$ gravity}

In this section we briefly introduce the $f(T)$ gravity theory and its covariant formulation. For more details we refer the reader to Ref. [19], where the notation, basic definition and proofs are presented, and to Ref. [67], where the formulation of the covariant version of $f(T)$ gravity theory is presented. For a pedagogical presentation of the basic geometrical setting of these theories, see Appendix J of Carroll's book [95].

We begin by recalling that the dynamical variables in $f(T)$ gravity theories are the tetrad fields, $\mathbf{h}_{A}\left(x^{\mu}\right)$, which is a set of four $(A=0, \ldots, 3)$ vector fields that define a local orthonormal Lorentz frame at every point $x^{\mu}$ of the space-time manifold. The vector fields $\mathbf{h}_{A}\left(x^{\mu}\right)$ are vectors in the tangent space at an arbitrary point $x^{\mu}$ of the space-time manifold. The space-time and the tangent space metrics are related by

$g_{\mu \nu}=h_{\mu}^{A} h_{v}^{B} \eta_{A B}$,

where $\eta_{A B}=\operatorname{diag}(1,-1,-1,-1)$ is the Minkowski metric of the tangent space at $x^{\mu}$. Here and in what follows, we use Greek letters to denote space-time coordinate indices, which are raised and lowered, respectively, with $g_{\mu \nu}$ and $g^{\mu \nu}$ and vary from 0 to 3 , whereas Latin upper case letters denote tetrad indices, which are lowered and raised with the Minkowski tensor $\eta_{A B}$ and $\eta^{A B}$. It follows from Eq. (1) that the relation between the frame components, $h_{A}^{\mu}$, and the coframe components, $h_{\mu}^{A}$, are given by

$h_{A}^{\mu} h_{v}^{A}=\delta_{v}^{\mu}$ and $h_{A}^{\mu} h_{\mu}^{B}=\delta_{A}^{B}$.
Under local Lorentz transformations at each point $x$ the tetrad fields transform as

$\mathbf{h}_{B} \longrightarrow \mathbf{h}_{A}^{\prime}=\Lambda_{A}^{B}(x) \mathbf{h}_{B}$

and the metric is left invariant, i.e.,

$\eta_{A B}^{\prime}=\Lambda_{A}^{C} \eta_{C D} \Lambda_{B}^{D}=\operatorname{diag}(1,-1,-1,-1)$.

Considering that at every point of the space-time besides the general coordinate transformations, $x^{\mu} \rightarrow x^{\mu^{\prime}}$, we have the freedom to perform a Lorentz transformation one finds a mixed transformation law as, for example,

$M_{B v}^{\prime A \mu}=\Lambda_{C}^{A} \frac{\partial x^{\mu^{\prime}}}{\partial x^{\lambda}} \Lambda_{B}^{D} \frac{\partial x^{\rho}}{\partial x^{\nu^{\prime}}} M_{D \rho}^{C \lambda} \cdot$

For differentiating geometrical objects as, e.g., $X_{B}^{A}$ we replace the ordinary connection by the spin connection, denoted by $\omega_{B \mu}^{A}$, and each Latin upper case index gets a factor of the spin connection according to

$\nabla_{\mu} X_{B}^{A}=\partial_{\mu} X_{B}^{A}+\omega^{A}{ }_{C \mu} X_{B}^{C}-\omega_{B \mu}^{C} X_{C}^{A}$.

Under local Lorentz transformations, $\Lambda_{B}^{A}(x)$, the spin connections transforms as

$\omega_{B \mu}^{\prime A}=\Lambda_{C}^{A} \omega_{D \mu}^{C} \Lambda_{B}^{D}+\Lambda_{C}^{A} \partial_{\mu} \Lambda_{B}^{C}$,

where $\Lambda_{B}{ }^{A}$ is the inverse of $\Lambda^{A}{ }_{B}$. The spin connection in teleparallel gravity is meant to represent only inertial or frame effects. This means that there exists a class of frames relative to which the spin connection vanishes, $\widetilde{\omega}_{D \mu}^{C}=0$. From this fact along with Eq. (7) one sees that in a general class of frames the spin connection takes the form [97]

$\omega_{B \mu}^{A}=\Lambda_{C}^{A} \partial_{\mu} \Lambda_{B}^{C}$.

In covariant $f(T)$ gravity theories, instead of the LeviCivita connection, one uses the Weitzenböck connection, which is given by

$\Gamma_{v \mu}^{\rho}=h_{A}^{\rho}\left(\partial_{\mu} h_{v}^{A}+\omega_{B \mu}^{A} h_{v}^{B}\right)$,

with the inverse relation

$\omega_{B \mu}^{A}=h_{\rho}^{A} \partial_{\mu} h_{B}^{\rho}+h_{\rho}^{A} \Gamma_{\nu \mu}^{\rho} h_{B}^{\nu} \equiv h_{\rho}^{A} \nabla_{\mu} h_{B}{ }^{\rho}$,

where $\nabla_{\mu}$ denotes the covariant derivative. An immediate consequence of this definition for the covariant derivative is that for the spin connection (8) there is no curvature but a nonzero torsion,

$T_{\mu \nu}^{\rho}=h_{A}^{\rho} T_{\mu \nu}^{A}=\Gamma_{\nu \mu}^{\rho}-\Gamma_{\mu \nu}^{\rho}$, 
where in terms of the tetrad fields, the torsion tensor is defined by [19]

$T_{\mu \nu}^{A} \equiv \partial_{\mu} h_{\nu}^{A}-\partial_{\nu} h_{\mu}^{A}+\omega_{B \mu}^{A} h_{\nu}^{B}-\omega_{B \nu}^{A} h_{\mu}^{B}$.

Now, if one further defines the so-called super-potential

$S_{A}^{\mu \nu} \equiv K_{A}^{\mu \nu}+h_{A}^{\mu} T_{\theta}^{\theta v}-h_{A}^{\nu} T_{\theta}^{\theta \mu}$,

where

$K_{A}^{\mu \nu} \equiv-\frac{1}{2}\left(T_{A}^{\mu \nu}-T_{A}^{\nu \mu}-T_{A}^{\mu \nu}\right)$

is the contorsion tensor, we can define the torsion scalar

$T \equiv \frac{1}{2} S_{A}^{\mu \nu} T_{\mu \nu}^{A}$

This scalar is used in the Lagragian density for the formulation of the covariant $f(T)$ gravity theory, whose action is defined by

$S=\int d^{4} x h\left[\frac{f(T)}{2 \kappa}+\mathscr{L}_{m}\right]$,

where $h=\operatorname{det}\left(h_{\mu}^{A}\right), \kappa=8 \pi G, f(T)$ is an arbitrary function of the torsion scalar $T$ and $\mathscr{L}_{m}$ is the Lagrangian density for the matter field. ${ }^{6}$

Varying the action (16) with respect to the vierbein and taking into account Eqs. (12)-(15) one obtains the following equations for the covariant $f(T)$ gravity:

$$
\begin{gathered}
h^{-1} f_{T} \partial_{\nu}\left(h S_{A}^{\mu \nu}\right)+f_{T T} S_{A}^{\mu \nu} \partial_{\nu} T-f_{T} h_{A}^{\lambda} T_{\nu \lambda}^{B} S_{B}^{\nu \mu} \\
+f_{T} \omega_{A \nu}^{B} S_{B}^{\nu \mu}+\frac{1}{2} f(T) h_{A}^{\mu}=\kappa h_{A}^{\nu} \Theta_{\nu}^{\mu},
\end{gathered}
$$

where $f_{T}=d f(T) / d T, f_{T T}=d^{2} f(T) / d T^{2}$, and $\Theta_{\nu}{ }^{\mu}$ is the energy-momentum tensor of the matter fields defined as

$\Theta_{v}^{\mu}=\left(-\frac{1}{h} \frac{\delta S_{m}}{\delta h_{\mu}^{A}}\right) h_{v}^{A}$,

where $S_{m}=\int d^{4} x h \mathscr{L}_{m}$ is the matter action.

It should be emphasized that, unlike the usual formulation of $f(T)$ gravity, where it is implicitly assumed that the spin connection $\omega_{B \mu}^{A}$ vanishes, in the derivation of the above field equations (17) a non-vanishing $\omega_{B \mu}^{A}$ is assumed from the outset. Actually, the presence of the nonzero spin connection term in the field equations (17) ensures the Lorentz covariance of Eqs. (17) (for details see Ref. [67]). The price for this is that now we have to figure out a way to determine

\footnotetext{
${ }^{6}$ In the particular case $f(T)=T$ one has the teleparallel equivalent of general relativity (TEGR).
}

the non-vanishing spin connection $\omega_{B \mu}^{A}$, a point that we shall discuss in the following.

The covariant field equations (17) depend not only on the vierbein but also on the non-vanishing spin connection $\omega_{B \mu}^{A} \cdot{ }^{7}$ Thus, solutions to the covariant field equations can be found if one establishes a procedure to suitably determine the spin connection $\omega_{B \mu}^{A}$. In what follows we shall briefly present a scheme figured out in Ref. [67] to determine $\omega_{B \mu}^{A}$. It consists in starting from the arbitrarily chosen initial tetrad field $h_{\rho}^{A}$, define a reference frame, $h_{(r) \mu}^{A}$, in which gravity is switched off, namely

$\left.h_{(r) \mu}^{A} \equiv h_{\mu}^{A}\right|_{\text {Grav } \longrightarrow 0}$.

In other words, when the gravity is switched off the metric

$g_{\mu \nu}=h_{(r) \mu}^{A} h_{(r) v}^{B} \eta_{A B}$

reduces to the Minkowski metric.

Following the procedure of Ref. [67], we then require the torsion to vanish for this reference tetrad, i.e. $T_{\mu \nu}^{A}\left(h_{(r) \mu}^{A}\right.$, $\left.\omega_{B \mu}^{A}\right)=0$. Thus, to have the Lorentz covariant field equations we take the spin connection for the field equations (17) as

$\omega_{B \mu}^{A}\left(h_{\mu}^{A}\right) \equiv \omega_{B \mu}^{A}\left(h_{(r) \mu}^{A}\right)$.

The underlying basic idea behind this choice of spin connection is that the torsion tensor (12) depends on both the tetrad and the spin connection. Thus, in general the torsion embodies the field strength of gravity along with inertial effects. However, there exists a choice of the spin connection for which the torsion captures only the strength of gravity. This spin connection is obtained by using the reference tetrad $h_{(r) \mu}^{A}$ as defined by (19) (or (20)), and then by defining $\omega_{B \mu}^{A}$ according to (21) (for more details see Ref. [67]).

In practice, this can be done by taking, in the general expression for the spin connection, (Eq. (1.60) of Ref. [19]),

$\omega_{B \mu}^{A}=\frac{1}{2} h_{\mu}^{C}\left[f_{B C}^{A}+T_{B C}^{A}+f_{C B}^{A}+T_{C B}^{A}-f_{B C}^{A}-T_{B C}^{A}\right]$,

where $\left[h_{A}, h_{B}\right]=f_{A B}^{C} h_{C}$, and the torsion $T_{B C}^{A}=$ $h_{B}^{\mu} h_{C}^{\nu} T_{\mu \nu}^{A}=0$ for the reference tetrad $h_{(r) \mu}^{A}$. In this way, one has

$\omega_{B \mu}^{A}=\frac{1}{2} h_{(r) \mu}^{C}\left[f_{B C}^{A}\left(h_{(r)}\right)+f_{C B}^{A}\left(h_{(r)}\right)-f_{B C}^{A}\left(h_{(r)}\right)\right]$,

\footnotetext{
$\overline{7}$ In the usual $f(T)$ gravity the field equations are differential equations for the tetrads only.
} 
where the structure coefficients $f_{B C}^{A}\left(h_{(r)}\right)$ are calculated from the following equation (Eq. (1.32) of Ref. [19]):

$f_{A B}^{C}=h_{A}^{\mu} h_{B}^{\nu}\left(\partial_{\nu} h_{\mu}^{C}-\partial_{\mu} h_{\nu}^{C}\right)$,

evaluated for the reference vierbein $h_{(r) \mu}^{A}$.

\section{Gödel-type geometries}

In this section we present the main properties of the homogeneous Gödel-type geometries, which we use in the next section. We begin by recalling that the Gödel solution to the general relativity field equations is a specific member of the large family of geometries, whose general form in cylindrical coordinates, $(r, \varphi, z)$, is [98]

$\mathrm{d} s^{2}=[\mathrm{d} t+H(r) \mathrm{d} \varphi]^{2}-D^{2}(r) \mathrm{d} \varphi^{2}-\mathrm{d} r^{2}-\mathrm{d} z^{2}$.

The necessary and sufficient conditions for the Gödeltype geometries (25) to be space-time homogeneous (SThomogeneous) are $[98,99]$

$$
\frac{H^{\prime}}{D}=2 \Omega \text { and } \frac{D^{\prime \prime}}{D}=m^{2},
$$

where the prime denotes the derivative with respect $r$, and the parameters $(\Omega, m)$ are constants such that $-\infty \leq m^{2} \leq$ $\infty$ and $\Omega^{2}>0$. The ST-homogeneity is ensured by the fact that Gödel-type space-times admit either a group $G_{5}$ or $G_{7}$ of isometries acting transitively on the whole spacetime $[99,100]$.

Another important property of Gödel-type geometries is the existence of an irreducible set of isometrically nonequivalent classes of ST-homogeneous Gödel-type, which are given as follows [98] (see also Ref. [101]).

i. Hyperbolic case, in which $m^{2}=$ const $>0$ and

$$
H=\frac{4 \Omega}{m^{2}} \sinh ^{2}\left(\frac{m r}{2}\right), \quad D=\frac{1}{m} \sinh (m r) .
$$

ii. Linear case, in which $m=0$ and

$$
H=\Omega r^{2}, \quad D=r .
$$

iii. Trigonometric case, where $m^{2}=$ const $\equiv-\mu^{2}<0$ and

$$
H=\frac{4 \Omega}{\mu^{2}} \sin ^{2}\left(\frac{\mu r}{2}\right), \quad D=\frac{1}{\mu} \sin (\mu r) .
$$

Accordingly, the ST-homogeneous Gödel-type metrics are characterized by the essential parameters $m^{2}$ and $\Omega$. Incidentally, the Gödel's solution of the Einstein equations is a particular member of the hyperbolic class $\left(m^{2}>0\right)$ in which $m^{2}=2 \Omega^{2}$.

To examine the causality features of the ST-homogeneous Gödel-type metrics we rewrite the line element (25) as

$\mathrm{d} s^{2}=\mathrm{d} t^{2}+2 H(r) \mathrm{d} t \mathrm{~d} \phi-\mathrm{d} r^{2}-G(r) \mathrm{d} \phi^{2}-\mathrm{d} z^{2}$,

where $G(r)=D^{2}(r)-H^{2}(r)$. In this form it is straightforward to show the existence of closed time-like curves (CTC), i.e. make explicit the violation of causality in SThomogeneous Gödel-type space-times. Indeed, from Eq. (30) one sees that the circles defined by $t, z, r=$ const with $r>0$ are closed time-like curves whenever $G(r)<0$ (the line element $\mathrm{d} s^{2}$ becomes space-like, and its integral curves are closed). Thus, for the hyperbolic class $\left(m^{2}>0\right)$ one finds that $G(r)=D^{2}(r)-H^{2}(r)$ is given by

$G(r)=\frac{4}{m^{2}} \sinh ^{2}\left(\frac{m r}{2}\right)\left[\left(1-\frac{4 \Omega^{2}}{m^{2}}\right) \sinh ^{2}\left(\frac{m r}{2}\right)+1\right]$.

Therefore for $0<m^{2}<4 \Omega^{2}$ there is a finite critical radius $r_{c}$ defined by $G(r)=0$ given by

$\sinh ^{2}\left(\frac{m r_{c}}{2}\right)=\left(\frac{4 \Omega^{2}}{m^{2}}-1\right)^{-1}$,

and such that $G(r)>0$ for $r<r_{c}$ and $G(r)<0$ for $r>r_{c}$. Therefore, the circles $t, r, z=$ const in the circular band with $r>r_{c}$ are CTCs. One particularly important case in the range $0<m^{2}<4 \Omega^{2}$ of the hyperbolic class is the Gödel metric $\left(m^{2}=2 \Omega^{2}\right)$, for the which there is a finite critical radius and therefore breakdown of causality in the form of CTCs. Another important Gödel-type metric in the hyperbolic family is defined by $m^{2}=4 \Omega^{2}$. Indeed, in this case from (32) one sees that the critical radius goes to infinity, $r_{c} \rightarrow \infty$, and therefore there is no violation of causality, since $G(r)>0$ for all $0<r<\infty$. An Einstein field equations solution of this specific type was found in Ref. [98].

For the linear family $(m=0)$ one easily finds that $G(r)=$ $D^{2}(r)-H^{2}(r)$ is given by

$G(r)=r^{2}(1-\Omega r)(1+\Omega r)$.

Similarly to the hyperbolic class, for this family there is a critical radius [ $G(r)=0$ ] given by $r_{c}=1 / \Omega$ such that for any radius $r>r_{c}$ the inequality $G(r)<0$ holds, and thus circles defined by $t, z, r=$ const are CTCs.

Finally, for the trigonometric class $m^{2}<0$ one finds that $G(r)$ reduces to

$G(r)=\frac{4}{\mu^{2}} \sin ^{2}\left(\frac{\mu r}{2}\right)\left[1-\left(1+\frac{4 \Omega^{2}}{\mu^{2}}\right) \sin ^{2}\left(\frac{\mu r}{2}\right)\right]$, 
but now, differently from the other two class, there is an infinite sequence of alternating causal [ $G(r)>0$ ] and noncausal $[G(r)<0]$ regions (circular bands) in the section $t, z, r=$ const (with $r>0$ ) without and with non-causal Gödel's circles, depending on the value of $r$. Thus, e.g., if $G(r)<0$ for a certain range $r_{1}<r<r_{2}$ non-causal circles exist, whereas for $r$ in the next circular region $r_{2}<r<$ $r_{3}$ for which $G(r)>0$ no such non-causal Gödel's circles exist [98, 101].

To close this section, we mention that throughout this work by non-causal and causal solutions we mean solutions with and without violation of causality of Gödel type, i.e., with and without the Gödel non-causal circles discussed above.

\section{Gödel-type solutions in the covariant $f(T)$ gravity}

The aim of this section is twofold. First, following the scheme devised in Ref. [67] we determine the spin connection associated with a Lorentz tetrad of Gödel-type space-time geometries (25), which allow for the field equations for these spacetimes in the covariant $f(T)$ gravity. Second, we take a combination of a perfect fluid with electromagnetic plus scalar fields as source, and we determine a general Gödel-type solution of the covariant $f(T)$ field equations; also we discuss its main important properties.

\subsection{Field equations}

At an arbitrary point Gödel-type space-time manifold we choose the following tetrad basis $\theta^{A}=h_{\mu}^{A} d x^{\mu}$ :

$\theta^{0}=\mathrm{d} t+H(r) \mathrm{d} \varphi, \quad \theta^{1}=\mathrm{d} r$,

$\theta^{2}=D(r) \mathrm{d} \varphi, \quad \theta^{3}=\mathrm{d} z$,

relative to which the Gödel-type line element (25) clearly takes the form

$d s^{2}=\eta_{A B} \theta^{A} \theta^{B}=\eta_{A B} h_{\mu}^{A} h_{\nu}^{B} d x^{\mu} d x^{\nu}$,

$\eta_{A B}=\operatorname{diag}(+1,-1,-1,-1)$, and the components of the tetrad fields $h_{\mu}^{A}$ and $h_{A}^{\mu}$ are given by

$h_{\mu}^{A}=\left(\begin{array}{cccc}1 & 0 & H & 0 \\ 0 & 1 & 0 & 0 \\ 0 & 0 & D & 0 \\ 0 & 0 & 0 & 1\end{array}\right)$ and $h_{A}^{\mu}=\left(\begin{array}{cccc}1 & 0 & -\frac{H}{D} & 0 \\ 0 & 1 & 0 & 0 \\ 0 & 0 & \frac{1}{D} & 0 \\ 0 & 0 & 0 & 1\end{array}\right)$.

From Eqs. (37) and (38) one sees that gravity is switched off when $H(r) \rightarrow 0$ and $D(r) \rightarrow r$, inasmuch as in this limit the tetrads reduce to the tetrads of the Minkowski space-time in cylindrical coordinates, namely $h_{(\mathrm{M}) \mu}^{A}=\operatorname{diag}(1,1, r, 1)$.
Thus, following the scheme devised in Ref. [67] the reference frame, $h_{(r) \mu}^{A}$, defined by

$\left.h_{(r) \mu}^{A} \equiv h_{\mu}^{A}\right|_{\text {Grav } \longrightarrow 0}$,

is given by

$h_{(r) \mu}^{A}=h_{(\mathrm{M}) \mu}^{A}=\operatorname{diag}(1,1, r, 1)$.

For this reference frame, by using Eq. (24), we obtain the following non-vanishing components of the anholonomy coefficients:

$f_{\hat{2} \hat{1}}^{\hat{2}}=-f_{\hat{1} \hat{2}}^{\hat{2}}=\frac{1}{r}$

and then, by using Eq. (23), one finds that the nonzero spin connection associated to the reference frame (40) is given by

$\omega_{\hat{1} 2}^{\hat{2}}=-\omega_{\hat{2} 2}^{\hat{1}}=1$,

where the hat over the numerical indices is used to denote that the corresponding digits are tetrad indices.

To have the Lorentz covariant field equations we take the spin $\omega_{B \mu}^{A}\left(h_{\mu}^{A}\right)=\omega_{B \mu}^{A}\left(h_{(r) \mu}^{A}\right)$. Thus, from Eqs. (38) and (42) the field equations (17) reduce, for Gödel-type space-times, to ${ }^{8}$

$\left[\frac{H T^{\prime}}{2 H^{\prime}}-\frac{D^{\prime \prime}}{D}+T\right] f_{T}+\left[\frac{H T}{H^{\prime}}-\frac{D^{\prime}}{D}\right] f_{T T} T^{\prime}+\frac{1}{D} f_{T T} T^{\prime}$
$+\frac{f}{2}=\kappa \Theta_{0}^{0}$
$\left[\frac{D^{2} T^{\prime}}{2 H^{\prime}}+H T\right] f_{T}+\frac{H^{\prime} T^{\prime}}{2} f_{T T}-\frac{H}{2} f=\kappa\left(\Theta_{2}^{0}-H \Theta_{0}^{0}\right)$,

$-T f_{T}+\frac{f}{2}=\kappa \Theta_{1}^{1}$,

$-\frac{T^{\prime}}{H^{\prime}}\left[\frac{f_{T}}{2}+T f_{T T}\right]=\kappa \Theta_{0}^{2}$,

$-T f_{T}+\frac{f}{2}=\kappa\left(\Theta_{2}^{2}-H \Theta_{0}^{2}\right)$,

$\frac{1}{D} f_{T T} T^{\prime}-\frac{D^{\prime}}{D} T^{\prime} f_{T T}-\frac{D^{\prime \prime}}{D} f_{T}+\frac{f}{2}=\kappa \Theta_{3}{ }^{3}$,

where we have used the fact that, from Eq. (15) along with Eqs. (13), (14) and (12), the torsion scalar is given by

$T=\frac{1}{2}\left(\frac{H^{\prime}}{D}\right)^{2}$.

\footnotetext{
8 We note that the additive term $D^{-1} f_{T T} T^{\prime}$ in equations (43) and (48) do not arise in the usual formulation of $f(T)$ gravity. For comparison we refer the reader to Ref. [102], where similar field equations were derived in the context of the usual $f(T)$ gravity.
} 
Since in this paper we are interested in ST-homogeneous Gödel-type space-times, the homogeneity conditions (26) hold, and therefore the torsion scalar is given by

$T=2 \Omega^{2}$

and the field equations (43) to (48) reduce to

$$
\begin{aligned}
& \left(2 \Omega^{2}-m^{2}\right) f_{T}+\frac{f}{2}=\kappa \Theta_{0}^{0}, \\
& 2 \Omega^{2} H f_{T}-\frac{H}{2} f=\kappa\left(\Theta_{2}^{0}-H \Theta_{0}^{0}\right), \\
& -2 \Omega^{2} f_{T}+\frac{f}{2}=\kappa \Theta_{1}{ }^{1}=\kappa \Theta_{2}^{2}, \\
& \Theta_{0}{ }^{2}=0, \\
& -m^{2} f_{T}+\frac{f}{2}=\kappa \Theta_{3}^{3} .
\end{aligned}
$$

Since the homogeneous Gödel-type metric (25) and tetrads $h_{\mu}^{A}$ [Eq.(38)] are determined by the parameters $m^{2}$ and $\Omega$, solving equations (51)-(55) for these parameters one finds

$$
\begin{aligned}
& m^{2}=\frac{\kappa}{f_{T}}\left(\Theta_{0}{ }^{0}+\Theta_{1}{ }^{1}-2 \Theta_{3}{ }^{3}\right), \\
& \Omega^{2}=\frac{\kappa}{2 f_{T}}\left(\Theta_{0}{ }^{0}-\Theta_{3}{ }^{3}\right),
\end{aligned}
$$

subject to the constraints

$$
\begin{aligned}
& f=2 \kappa\left(\Theta_{0}^{0}+\Theta_{1}{ }^{1}-\Theta_{3}^{3}\right), \\
& \Theta_{2}{ }^{0}-H\left(\Theta_{0}{ }^{0}-\Theta_{1}{ }^{1}\right)=\Theta_{0}{ }^{2}=0,
\end{aligned}
$$

where the functions $f$ and $f_{T}$ are evaluated at $T=2 \Omega^{2}$, and where we assume that $f_{T}>0$ to ensure that the effective Newton constant does not change its sign.

It should be emphasized that in the field equations (56) (59) no specific energy-momentum tensor have been used. In the next subsection, a concrete matter content will be considered in the search for ST-homogeneous Gödel-type solutions of the covariant $f(T)$ field equations. To this end, we shall take a combination of a perfect fluid with scalar field along with an electromagnetic field. Clearly, the scalar and electromagnetic field sources have to fulfill, respectively, the Klein-Gordon and Maxwell equations in the curved Gödeltype background geometry.

\subsection{Solutions}

In this section we discuss ST-homogeneous Gödel-type solutions of the covariant $f(T)$ gravity for specific matter sources. To this end, we take a combination of scalar and electromagnetic fields with a perfect fluid as a matter source, find a general solution, and examine several special Gödel-type solutions. Thus, in the local frame defined by
Eqs. (35) and (36) the energy-momentum tensor $\Theta_{A B}$ for the combined fields has the form

$\Theta_{A B}=\stackrel{\text { pf }}{\Theta}_{A B}+\stackrel{\text { ff }}{\Theta}_{A B}+\stackrel{\text { ef }}{\Theta}_{A B}$,

where $\stackrel{\text { pf }}{\Theta}_{A B}, \stackrel{\text { ff }}{\Theta}_{A B}$ and $\stackrel{\text { ef }}{\Theta}_{A B}$ are, respectively, the energymomentum tensors of a perfect fluid, a scalar field and an electromagnetic field, which are given by

pf

$\Theta_{A B}=(\rho+p) u_{A} u_{B}-p \eta_{A B}$,

$\stackrel{\text { sf }}{\Theta}_{A B}=\nabla_{A} \phi \nabla_{B} \phi-\eta_{A B}\left[\frac{1}{2} \eta^{C D} \nabla_{C} \phi \nabla_{D} \phi\right]$,

$\stackrel{\text { ef }}{\Theta}_{A B}=-F_{A}{ }^{C} F_{B C}+\frac{1}{4} \eta_{A B} F_{C D} F^{C D}$,

where $u_{A}=\delta_{A}^{0}$ is the four-velocity, $\rho$ and $p$ are the energy density and pressure of the perfect fluid, subject to the weak energy condition (WEC) $\rho>0$ and $\rho+p>0$, and where $\nabla_{A} \phi$ denotes the covariant derivatives relative to the local basis $\theta^{A}=h_{\alpha}^{A} d x^{\alpha}$.

The massless scalar field $\phi$ fulfills the Klein-Gordon equation,

$\square \phi=\eta^{A B} \nabla_{A} \nabla_{B} \phi=0$,

a solution of which can be written as $\phi=\phi(z)=s\left(z-z_{0}\right)$, where $s, z_{0}=$ const [98]. Thus, the non-vanishing components of the energy-moment tensor are

$\stackrel{\mathbf{s f}}{\Theta}_{\hat{0} \hat{0}}=-\stackrel{\text { ff }}{\Theta}_{\hat{1} \hat{1}}=-\stackrel{\text { ff }}{\Theta}_{\hat{2} \hat{2}}=\stackrel{\text { sf }}{\Theta}_{\hat{3} \hat{3}}=\frac{s^{2}}{2}$.

The electromagnetic field $F_{A B}$ satisfies the source-free Maxwell equations

$\nabla_{A} F^{A B}=0$ and $\nabla_{A}\left(\star F^{A B}\right)=0$,

where $\star F^{A B}=\frac{1}{2} \varepsilon^{A B C D} F_{C D}$. Following Ref. [98], it can be shown that the electromagnetic field tensor $F_{A B}$ given by

$F_{\hat{0} \hat{3}}=-F_{\hat{3} \hat{0}}=e \sin \left[2 \Omega\left(z-z_{0}\right)\right]$,

$F_{\hat{1} \hat{2}}=-F_{\hat{2} \hat{1}}=e \cos \left[2 \Omega\left(z-z_{0}\right)\right]$,

where $e$ is constant, satisfies the Maxwell equations (66). Hence, the non-vanishing components of the associated energy-momentum tensor $\Theta_{A B}=F_{A}{ }^{C} F_{B C}+\frac{1}{4} \eta_{A B} F^{C D} F_{C D}$ are given by

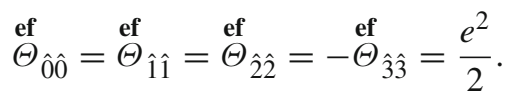

Thus, taking into account Eqs. (61), (65) and (69), one sees that for the combined-field matter source we have (60), 
and therefore from Eqs. (56), (57) and (58) one sees that a general solution to the field equations can be written as ${ }^{9}$

$$
\begin{aligned}
& m^{2}=\frac{\kappa}{f_{T}}\left(\rho+p+2 s^{2}-e^{2}\right), \\
& \Omega^{2}=\frac{\kappa}{2 f_{T}}\left(\rho+p+s^{2}\right), \\
& f=\kappa\left(2 \rho+3 s^{2}-e^{2}\right),
\end{aligned}
$$

and Eqs. (59) are identically satisfied.

Since ST-homogeneous Gödel-type geometries (25) and tetrads (38) are characterized by the two essential parameters $m^{2}$ and $\Omega$, Eqs. (70) and (71) along with the constraint equation (72) make explicit how the covariant $f(T)$ gravity version specifies a pair of parameters $\left(\mathrm{m}^{2}, \Omega^{2}\right)$, and therefore determines a general ST-homogeneous Gödel-type solution for the combined-fields matter source (60). In the remainder of this section we discuss the most significant special cases of this general solution and some important characteristics of these Gödel-type solutions in the covariant $f(T)$ gravity.

A first important point regarding the above general solution for the combined field is that from Eq. (70) we clearly have solutions in the hyperbolic (27), linear (28) or trigonometric (29) families of Gödel-type space-times.

Since $f_{T}>0$, solutions are in the hyperbolic class when $\rho+p+2 s^{2}-e^{2}>0$. We note, however, that from Eqs. (70) and (71) there is an upper bound for $m^{2}$ since

$m^{2}-4 \Omega^{2}=-\frac{\kappa}{f_{T}}\left(\rho+p+e^{2}\right) \leq 0$.

From Eqs. (70) and (71) it is easy to show that the limiting bound solution $m^{2}=4 \Omega^{2}$ takes place for single scalar field of amplitude $s$ as the matter source. Besides, according to Eq. (32) of Sect. 3 the critical radius goes to infinity, $r_{c} \rightarrow \infty$, and therefore there is no violation of causality for this particular solution. Apart from this particular case, from Eq. (32) along with (70) and (71) one sees that for all solutions in the hyperbolic class with $m^{2}<4 \Omega^{2}$ there is a finite critical radius, beyond which causality is violated, given by

$r_{c}=\frac{2\left(f_{T}\right)^{\frac{1}{2}} \sinh ^{-1}\left(\sqrt{\frac{\rho+p+2 s^{2}-e^{2}}{\rho+p+e^{2}}}\right)}{\left[\kappa\left(\rho+p+2 s^{2}-e^{2}\right)\right]^{1 / 2}}$.

As one would expect from the outset this critical radius depends upon the $f(T)$ gravity theory and the matter content.

A particularly important case in the hyperbolic family is the Gödel space-time, in which $m^{2}=2 \Omega^{2}$. From Eqs. (70) and (71) there are two possible matter sources that give rise to a Gödel solution in covariant $f(T)$ gravity. First, a perfect

\footnotetext{
${ }^{9}$ Clearly we have used the fact that the coordinate components of the energy-momentum tensor are given by $\Theta_{\mu}^{\nu}=h_{B}^{\nu} h_{\mu}^{A} \Theta_{A}^{B}$.
}

fluid of density $\rho$ and pressure $p$ subject to the WEC and with $f_{T}>0$. In this case $s^{2}=e^{2}=0$ and therefore $m^{2}=2 \Omega^{2}$ holds. The fact that all perfect-fluid solutions subject to the WEC are isometric to Gödel geometry extends the Bampi and Zordan theorem [96] demonstrated in the framework of GR to the context of covariant $f(T)$ gravity with $f_{T}>0$. Second, for a simple combination of the above scalar and electromagnetic fields $(\rho=p=0)$ from Eqs. (70) and (71) one has

$m^{2}-2 \Omega^{2}=\frac{\kappa}{f_{T}}\left(s^{2}-e^{2}\right)$.

Therefore, for $s^{2}=e^{2} \neq 0$ Gödel geometry is again recovered.

Regarding the linear and trigonometric families of Gödeltype space-times, from Eq. (70) we clearly see that to have either one of these classes the existence of the electromagnetic field component is required. The solutions in the linear class $\left(m^{2}=0\right)$ are obtained when $\rho+p+2 s^{2}-e^{2}=0$. Thus, for example, a simple combination of the above scalar and electromagnetic fields such that $e^{2}=2 s^{2}$ gives rise to a solution in the linear class. Since $\rho+p>0$ from WEC, in general, solutions in the linear class for the combined fields source arise only when $e^{2} \geq 2 s^{2}$. According to Sect. 3 and similarly to the hyperbolic class, for solutions belonging to the linear family there is a critical radius $r_{c}=1 / \Omega$ such that for any $r>r_{c}$ the Gödel circles (CTC) defined by $t, z, r=$ const arise.

Finally, solutions in the trigonometric class $\left(m^{2}=-\mu^{2}<\right.$ 0 ) arise when $\rho+p+2 s^{2}-e^{2}<0$. Very simple examples of solutions in this class are brought about when $\rho=p=0$ and $e^{2}>2 s^{2}$, but clearly we can have solutions with all the above combined-field matter components. As for the violation of causality, according to Sect. 3 for the trigonometric class there is an infinite sequence of alternating causal and noncausal circular bands in the section $t, z, r=$ const, $r>0$, with and without non-causal Gödel circles, depending on whether $G(r)<0$ or $G(r)>0$.

\section{Concluding remarks}

Despite the undeniable success of the general relativity theory, there has been a great deal of recent work on the so-called modified gravity theories. In the cosmological modeling this is motivated by the fact that these modified theories furnish an alternative way to account for the recent expansion of the Universe with no need to invoke the dark energy matter component. Several features of a family of these modified gravity theories, known as $f(T)$ gravity, have been discussed recently in a number of articles. Despite this noticeable interest in this new gravitational theory, it appears to have been overlooked in the recent literature that the $f(T)$ field equa- 
tions are not invariant under local Lorentz transformations, unless the $f(T)$ is a linear function of $T$, which is the teleparallel equivalent of general relativity (TEGR). This means that apart from the TEGR, different choices of the Lorentz tetrads give rise to different field equations. Relaxing a particular choice of the spin connection often made implicitly in the literature a locally Lorentz covariant $f(T)$ gravity theory has been devised recently [67].

Well-behaved chronology and causality are so essential in the special relativity theory that they are simply incorporated into the theory from its bare formulation. General relativity (GR) inherits locally the chronology and causality features of special relativity. On a non-local scale, however, important differences emerge and Einstein's field equations admit solutions to its field equations with closed time-like world lines, despite its local invariance under Lorentz transformations, which ensures locally the validity of the causality.

Even though different choices of local Lorentzian tetrads leave the metric invariant, suggesting at first sight a welldefined chronology and well-behaved causal structure, in the standard formulation of $f(T)$ gravity all different Lorentz tetrads give rise to different field equations, and therefore represent, in general, a different theory. Hence, for a given (fixed) Lorentz frame, which determines a fixed set of field equations (a fixed theory), there is no Lorentz transformation freedom. This means that unlike general relativity, a $f(T)$ gravity does not inherit locally the chronology and the wellbehaved causal structure of special relativity.

This local causality problem seems to have been overcome in Lorentz covariant $f(T)$ gravity [67], since in the new formulation of $f(T)$ theory the Lorentz transformations do not change the metric nor the field equations. The non-local question, however, is left open, and violation of causality may arise in the context of the covariant $f(T)$ gravity theory.

Since homogeneous Gödel-type geometries necessarily lead to the existence of closed time-like circles-an explicit manifestation of violation of causality on non-local scalea plausible way to answer this question is by investigating whether the covariant $f(T)$ gravity theories permit Gödeltype solutions. Furthermore, if gravity is to be governed by a covariant $f(T)$ theory there are a number of issues that ought to be examined in its context, including the question as to whether these theories permit Gödel-type solutions, or would remedy this causality problem by ruling them out.

In this paper, to look into the potentialities, difficulties and limitations of the covariant $f(T)$ gravity theories, we have addressed one of these questions and examined whether these theories admit homogeneous Gödel-type solutions for a number of matter sources. We have taken a combination of a perfect fluid with electromagnetic plus scalar fields as a matter source, and we determined a general Gödel-type solution. This general solution contains special solutions whose essential parameter, $m^{2}$, can take the sign that defines any class of homogeneous Gödel-type geometries, namely the hyperbolic family $\left(m^{2}>0\right)$, the trigonometric class $\left(m^{2}<0\right)$ and the linear class $(m=0)$. We have found that solutions in the trigonometric and linear classes are permitted only for the combined matter sources that include an electromagnetic field matter component. We have extended to the context of covariant $f(T)$ gravity the so-called Bampi-Zordan theorem, which ensures that any perfect-fluid ST-homogeneous Gödel-type solution in this theory $f(T)$ gravity is isometric to the Gödel metric and therefore defines the same set of Gödel tetrads $h_{A}^{\mu}$ up to a Lorentz transformation. We have also shown that the single massless scalar field generates the only ST-homogeneous Gödel-type solution with no closed time-like curves.

To conclude, we underline that it emerges from our results that even though the covariant $f(T)$ gravity restores local Lorentz covariance of the field equations and the validity of the causality principle locally, the bare existence of the Gödel-type solutions makes apparent that this covariant formulation of $f(T)$ gravity does not avoid non-local violation of causality in the form of the closed time-like curves that are permitted in general relativity.

Acknowledgements M.J. Rebouças acknowledges the support of FAPERJ under a CNE E-26/102.328/2017 grant and also thanks CNPq for the grant under which this work was carried out. G. Otalora acknowledges DI-VRIEA for financial support through Proyecto Postdoctorado 2017 VRIEA-PUCV. We are very grateful to A.F.F. Teixeira for indicating important omissions and misprints.

Open Access This article is distributed under the terms of the Creative Commons Attribution 4.0 International License (http://creativecomm ons.org/licenses/by/4.0/), which permits unrestricted use, distribution, and reproduction in any medium, provided you give appropriate credit to the original author(s) and the source, provide a link to the Creative Commons license, and indicate if changes were made. Funded by SCOAP ${ }^{3}$.

\section{References}

1. S. Capozziello, M. Francaviglia, Extended theories of gravity and their cosmological and astrophysical applications. Gen. Relat. Gravit. 40, 357 (2007)

2. A. De Felice, S. Tsujikawa, $f(R)$ theories. Living Rev. Rel. 13, 3 (2010)

3. T.P. Sotiriou, V. Faraoni, $f(R)$ theories of gravity. Rev. Mod. Phys 82, 451 (2010)

4. S. Nojiri, S.D. Odintsov, Unified cosmic history in modified gravity: from $F(R)$ theory to Lorentz non-invariant models. Phys. Rep. 505, $59(2011)$

5. G.J. Olmo, Palatini approach to modified gravity: $f(R)$ theories and beyond. Int. J. Mod. Phys. D 20, 413 (2011)

6. S. Capozziello, M. De Laurentis, Extended theories of gravity. Phys. Rep. 509, 167 (2011)

7. S. Capozziello, V. Faraoni, Beyond Einstein Gravity, Fundamental Theories of Physics, vol. 170 (Springer, Dordrecht, 2011)

8. G.R. Bengochea, R. Ferraro, Dark torsion as the cosmic speed-up. Phys. Rev. D 79, 124019 (2009) 
9. E.V. Linder, Einstein's other gravity and the acceleration of the universe. Phys. Rev. D 81, 127301 (2010). Erratum:[Phys. Rev. D 82, 109902 (2010)]

10. R. Myrzakulov, Accelerating universe from $F(T)$ gravity. Eur. Phys. J. C 71, 1752 (2011)

11. Y.F. Cai, S. Capozziello, M. De Laurentis, E.N. Saridakis, $f(T)$ teleparallel gravity and cosmology. Rept. Prog. Phys. 79, 106901 (2016)

12. A. Einstein, Sitzungsber. Preuss. Akad. Wiss. Phys. Math. K1. 217-224 (1928)

13. A. Unzicker, T. Case, Translation of Einstein's attempt of a unified field theory with teleparallelism. arXiv:physics/0503046

14. A. Einstein, Math. Ann. 102, 685 (1930)

15. C. Pellegrini, J. Plebański, K. Dan, Vidensk. Selsk. Mat. Fys. Skr. 2, 2 (1962)

16. C. Møller, K. Dan, Vidensk. Selsk. Mat. Fys. Skr. 89, 13 (1978)

17. K. Hayashi, T. Nakano, Extended translation invariance and associated gauge fields. Prog. Theor. Phys. 38, 491 (1967)

18. K. Hayashi, T. Shirafuji, New general relativity. Phys. Rev. D 19, 3524 (1979); Addendum. Phys. Rev. D 24, 3312 (1982)

19. R. Aldrovandi, J.G. Pereira, Teleparallel Gravity: An Introduction (Springer, Dordrecht, 2013)

20. V.C. de Andrade, L.C.T. Guillen, J.G. Pereira, Gravitational energy momentum density in teleparallel gravity. Phys. Rev. Lett. 84, $4533(2000)$

21. L. Iorio, E.N. Saridakis, Solar system constraints on $f(T)$ gravity. Mon. Not. Roy. Astron. Soc. 427, 1555 (2012)

22. L. Iorio, N. Radicella, M.L. Ruggiero, Constraining $f(T)$ gravity in the solar system. JCAP 1508, 021 (2015)

23. G. Farrugia, J.L. Said, M.L. Ruggiero, Solar system tests in $f(T)$ gravity. Phys. Rev. D 93, 104034 (2016)

24. G.R. Bengochea, Observational information for $f(T)$ theories and Dark Torsion. Phys. Lett. B 695, 405 (2011)

25. H. Wei, X.P. Ma, H.Y. Qi, $f(T)$ theories and varying fine structure constant. Phys. Lett. B 703, 74 (2011)

26. S. Capozziello, O. Luongo, E.N. Saridakis, Transition redshift in $f(T)$ cosmology and observational constraints. Phys. Rev. D 91, 124037 (2015)

27. V.K. Oikonomou, E.N. Saridakis, $f(T)$ gravitational baryogenesis. Phys. Rev. D 94, 124005 (2016)

28. R.C. Nunes, S. Pan, E.N. Saridakis, New observational constraints on $f(T)$ gravity from cosmic chronometers. JCAP 1608, 011 (2016)

29. J.B. Dent, S. Dutta, E.N. Saridakis, $f(T)$ gravity mimicking dynamical dark energy. Background and perturbation analysis. JCAP 1101, 009 (2011)

30. R. Zheng, Q.G. Huang, Growth factor in $f(T)$ gravity. JCAP 1103, $002(2011)$

31. K. Izumi, Y.C. Ong, Cosmological perturbation in $f(T)$ gravity revisited. JCAP 1306, 029 (2013)

32. B. Li, T.P. Sotiriou, J.D. Barrow, Large-scale structure in $f(T)$ gravity. Phys. Rev. D 83, 104017 (2011)

33. S. Basilakos, Linear growth in power law $f(T)$ gravity. Phys. Rev. D 93, 083007 (2016)

34. T. Wang, Static solutions with spherical symmetry in $f(T)$ theories. Phys. Rev. D 84, 024042 (2011)

35. K. Atazadeh, M. Mousavi, Vacuum spherically symmetric solutions in $f(T)$ gravity. Eur. Phys. J. C 73, 2272 (2013)

36. M.L. Ruggiero, N. Radicella, Weak-field spherically symmetric solutions in $f(T)$ gravity. Phys. Rev. D 91, 104014 (2015)

37. C.G. Böhmer, A. Mussa, N. Tamanini, Existence of relativistic stars in $f(T)$ gravity. Class. Quant. Grav. 28, 245020 (2011)

38. S. Capozziello, V.F. Cardone, H. Farajollahi, A. Ravanpak, Cosmography in $f(T)$-gravity. Phys. Rev. D 84, 043527 (2011)

39. D. Liu, M.J. Rebouças, Energy conditions bounds on $f(T)$ gravity. Phys. Rev. D 86, 083515 (2012)
40. R. Yang, New types of $f(T)$ gravity. Eur. Phys. J. C 71, 1797 (2011)

41. S.H. Chen, J.B. Dent, S. Dutta, E.N. Saridakis, Cosmological perturbations in $f(T)$ gravity. Phys. Rev. D 83, 023508 (2011)

42. R. Myrzakulov, $\mathrm{F}(\mathrm{T})$ gravity and k-essence. Gen. Relat. Gravit. 44, 3059 (2012)

43. K. Karami, A. Abdolmaleki, $f(T)$ modified teleparallel gravity models as an alternative for holographic and new agegraphic dark energy models. Res. Astron. Astrophys. 13, 757 (2013)

44. Y. Cai, S. Chen, J.B. Dent, S. Dutta, E.N. Saridakis, Matter Bounce Cosmology with the $f(T)$ Gravity. Class. Quant. Gravit. 28, 215011 (2011)

45. M. Li, R. Miao, Y. Miao, Degrees of freedom of $f(T)$ gravity. JHEP 1107, 108 (2011)

46. X. Meng, Y. Wang, Birkhoff's theorem in the $f(T)$ gravity. Eur. Phys. J. C 71, 1755 (2011)

47. H. Dong, Y.B. Wang, X.H. Meng, Extended Birkhoff's theorem in the $f(T)$ gravity. Eur. Phys. J. C 72, 2002 (2012)

48. C.G. Böhmer, A. Mussa, N. Tamanini, Existence of relativistic stars in $f(T)$ gravity. Class. Quant. Gravit. 28, 245020 (2011)

49. C.Q. Geng, C. Lee, E.N. Saridakis, Y. Wu, Teleparallel dark energy. Phys. Lett. B 704, 384 (2011)

50. K. Bamba, C.Q. Geng, Thermodynamics of cosmological horizons in $f(T)$ gravity. JCAP 1111, 008 (2011)

51. R. Ferraro, F. Fiorini, Spherically symmetric static spacetimes in vacuum $f(T)$ gravity. Phys. Rev. D 84, 083518 (2011)

52. R. Ferraro, F. Fiorini, Non trivial frames for $f(T)$ theories of gravity and beyond. Phys. Lett. B 702, 75 (2011)

53. H. Wei, Dynamics of teleparallel dark energy. Phys. Lett. B 712, 430 (2012)

54. Y. Wu, C.Q. Geng, Primordial fluctuations within teleparallelism. Phys. Rev. D 86, 104058 (2012)

55. C.G. Böhmer, T. Harko, F.S.N. Lobo, Wormhole geometries in modified teleparallel gravity and the energy conditions. Phys. Rev. D 85, 044033 (2012)

56. K. Atazadeh, F. Darabi, $f(T)$ cosmology via Noether symmetry. Eur. Phys. J. C 72, 2016 (2012)

57. K. Karami, A. Abdolmaleki, Generalized second law of thermodynamics in $f(T)$-gravity. JCAP 04, 007 (2012)

58. K. Bamba, G.G.L. Nashed, W. El Hanafy, S.K. Ibraheem, Bounce inflation in $f(T)$ cosmology: a unified inflaton-quintessence field. Phys. Rev. D 94, 083513 (2016)

59. K. Bamba, R. Myrzakulov, S. Nojiri, S.D. Odintsov, Reconstruction of $f(T)$ gravity: Rip cosmology, finite-time future singularities and thermodynamics. Phys. Rev. D 85, 104036 (2012)

60. K. Bamba, S.D. Odintsov, D. Saez-Gomez, Conformal symmetry and accelerating cosmology in teleparallel gravity. Phys. Rev. D 88, 084042 (2013)

61. K. Bamba, S. Nojiri, S.D. Odintsov, Trace-anomaly driven inflation in $f(T)$ gravity and in minimal massive bigravity. Phys. Lett. B 731, 257 (2014)

62. B. Li, T.P. Sotiriou, J.D. Barrow, $f(T)$ gravity and local Lorentz invariance. Phys. Rev. D 83, 064035 (2011)

63. R.X. Miao, M. Li, Y.G. Miao, Violation of the first law of black hole thermodynamics in $f(T)$ gravity. JCAP 1111, 033 (2011)

64. R. Zheng, Q. Huang, Growth factor in $f(T)$ gravity. JCAP 1103, $002(2011)$

65. T.P. Sotiriou, B. Li, J.D. Barrow, Generalizations of teleparallel gravity and local Lorentz symmetry. Phys. Rev. D 83, 104030 (2011)

66. N. Tamanini, C.G. Böhmer, Good and bad tetrads in $f(T)$ gravity. Phys. Rev. D 86, 044009 (2012)

67. M. Krššák, E.N. Saridakis, The covariant formulation of $f(T)$ gravity. Class. Quant. Gravit. 33, 115009 (2016) 
68. K. Gödel, An Example of a new type of cosmological solutions of Einstein's field equations of graviation. Rev. Mod. Phys. 21, 447 (1949)

69. M.M. Som, A.K. Raychaudhuri, Cylindrically symmetric charged dust distributions in rigid rotation in general relativity. Proc. Roy. Soc. Lond. A 304, 81 (1968)

70. M.J. Rebouças, A rotating universe with violation of causality. Phys. Lett. A 70, 161 (1979)

71. A.K. Raychaudhuri, S.N. Guha, Thakurta, Homogeneous spacetimes of the Gödel-type. Phys. Rev. D 22, 802 (1980)

72. M.J. Rebouças, A.F.F. Teixeira, Features of a relativistic spacetime with seven isometries. Phys. Rev. D 34, 2985 (1986)

73. F.M. Paiva, M.J. Rebouças, A.F.F. Teixeira, Time travel in the homogeneous Som-raychaudhuri universe. Phys. Lett. A 126, 168 (1987)

74. A. Krasiński, Rotating dust solutions of einsteins equations with 3dimensinal symmetry groups-III- all killing fields linearly independent of U(Alpha) And W(Alpha). J. Math. Phys. 39, 2148 (1998)

75. J.D. Barrow, C.G. Tsagas, Dynamics and stability of the Gödel universe. Class. Quant. Gravit. 21, 1773 (2004)

76. M.P. Dabrowski, J. Garecki, Energy momentum and angular momentum of Gödel universes. Phys. Rev. D 70, 043511 (2004)

77. J.D. Barrow, M.P. Dabrowski, Gödel universes in string theory. Phys. Rev. D 58, 103502 (1998)

78. J.E. Aman, J.B. Fonseca-Neto, M.A.H. MacCallum, M.J. Rebouças, Riemann-Cartan spacetimes of Gödel-type. Class. Quant. Gravit. 15, 1089 (1998)

79. M.J. Rebouças, A.F.F. Teixeira, Riemannian space-times of Gödel type in five dimensions. J. Math. Phys. 39, 2180 (1998)

80. M.J. Rebouças, A.F.F. Teixeira, Int. J. Mod. Phys. A 13, 3181 (1998)

81. P. Kanti, C.E. Vayonakis, Gödel type universes in string inspired charged gravity. Phys. Rev. D 60, 103519 (1999)

82. H.L. Carrion, M.J. Rebouças, A.F.F. Teixeira, Gödel-type spacetimes in induced matter gravity theory. J. Math. Phys. 40, 4011 (1999)

83. E.K. Boyda, S. Ganguli, P. Horava, U. Varadarajan, Holographic protection of chronology in universes of the Gödel type. Phys. Rev. D 67, 106003 (2003)

84. J.D. Barrow, C.G. Tsagas, The Gödel brane. Phys. Rev. D 69, 064007 (2004)
85. M. Banados, G. Barnich, G. Compere, A. Gomberoff, Threedimensional origin of Gödel spacetimes and black holes. Phys. Rev. D 73, 044006 (2006)

86. D. Astefanesei, R.B. Mann, E. Radu, Nut charged spacetimes and closed timelike curves on the boundary. JHEP 01, 049 (2005)

87. M.J. Rebouças, J. Santos, Gödel-type universes in $f(R)$ gravity. Phys. Rev. D 80, 063009 (2009)

88. J. Santos, M.J. Rebouças, T.B.R.F. Oliveira, Gödel-type universes in Palatini $f(R)$ gravity. Phys. Rev. D 81, 123017 (2010)

89. P.J. Porfírio, J.B. Fonseca-Neto, J.R. Nascimento, AYu. Petrov, J. Ricardo, A.F. Santos, Chern-Simons modified gravity and closed timelike curves. Phys. Rev. D 94, 044044 (2016)

90. J.R. Nascimento, AYu. Petrov, P.J. Porfirio, A.F. Santos, Gödeltype universes in Brans-Dicke theory. Phys. Lett. B 762, 96 (2016)

91. J.Santos, M.J. Rebouças, T.B.R.F. Oliveira, A.F.F. Teixeira, Homogeneous Gödel-type solutions in hybrid metric-Palatini gravity. arXiv:1611.03985 [gr-qc]

92. Y.C. Ong, K. Izumi, J.M. Nester, P. Chen, Problems with propagation and time evolution in $f(T)$ gravity. Phys. Rev. D 88, 024019 (2013)

93. K. Je-An, Y.C.Ong Gu, Acausality and nonunique evolution in generalized teleparallel gravity. Phys. Rev. D 89, 084025 (2014)

94. P. Chen, K. Izumi, J.M. Nester, Y.C. Ong, Remnant symmetry, propagation, and evolution in $f(T)$ gravity. Phys. Rev. D 91, 064003 (2015)

95. S. Carroll, Spacetime and Geometry: An Introduction to General Relativity (Addison Wesley, New York, 2004)

96. F. Bampi, C. Zordan, Gen. Rel. Grav. 9, 393 (1978)

97. M. Krššák, J.G. Pereira, Spin connection and renormalization of teleparallel action. Eur. Phys. J. C 75, 519 (2015)

98. M.J. Rebouças, J. Tiomno, On the homogeneity of riemannian space-times of Gödel type. Phys. Rev. D 28, 1251 (1983)

99. M.J. Rebouças, J.E. Aman, Computer-aided study of a class of Riemannian space-times. J. Math. Phys. 28, 888 (1987)

100. A.F.F. Teixeira, M.J. Rebouças, J.E. Aman, Isometries of homogeneous Gödel-type spacetimes. Phys. Rev. D 32, 3309 (1985)

101. J.B. Fonseca-Neto, A.Y. Petrov, M.J. Rebouças, Gödel-type universes and chronology protection in Horava-Lifshitz gravity. Phys. Lett. B 725, 412 (2013)

102. D. Liu, P. Wu, H. Yu, Gödel-type universes in $f(T)$ gravity. Int. J. Mod. Phys. D 21, 1250074 (2012) 